\title{
The application of adult traumatic brain injury models in a pediatric cohort
}

\author{
Adam M. H. Young, MRCS, ${ }^{1}$ Mathew R. Guilfoyle, MRCS, ${ }^{1}$ Helen Fernandes, MD, FRCS, ${ }^{1}$ \\ Matthew R. Garnett, DM, FRCS, ${ }^{1}$ Shruti Agrawal, FRCPCH, FFICM, ${ }^{2}$ and \\ Peter J. Hutchinson, PhD, FRCS ${ }^{1}$
}

\begin{abstract}
'Division of Academic Neurosurgery, Department of Clinical Neurosciences, and 'Department of Paediatric Intensive Care, Addenbrooke's Hospital, University of Cambridge, United Kingdom
\end{abstract}

\begin{abstract}
OBJECTIVE There is increasing interest in the use of predictive models of outcome in adult head injury. Two international models have been identified to be reliable modalities for predicting outcome: the Corticosteroid Randomisation After Significant Head Injury (CRASH) model, and the International Mission on Prognosis and Analysis of randomized Controlled Trials in TBI (IMPACT) model. However, these models are designed only to identify outcomes in adult populations.

METHODS A retrospective analysis was performed on pediatric patients with severe traumatic brain injury (TBI) admitted to the pediatric intensive care unit (PICU) of Addenbrooke's Hospital between January 2009 and December 2013. The individual risk of 14-day mortality was calculated using the CRASH-Basic and -CT models, and the risk of 6-month mortality calculated using the IMPACT-Core and -Extended (including CT findings) models. Model accuracy was determined by standardized mortality ratio (SMtR; observed/expected deaths), discrimination was evaluated as the area under the receiver operating curve (AUROC), and calibration assessed using the Hosmer-Lemeshow $\chi^{2}$ test.

RESULTS Ninety-four patients with an average age of 7.3 years were admitted to the PICU with a TBI. The mortality rate was $12.7 \%$ at 14 days and at 6 months. For the CRASH-Basic model, the SMtR was 1.42 and both calibration $\left(\chi^{2}=\right.$ $6.1, p=0.64$ ) and discrimination (AUROC $=0.92$ ) were good. For the IMPACT-Core model, the SMtR was 1.03 and the model was also well calibrated $\left(\chi^{2}=8.99, p=0.34\right.$ ) and had good discrimination (AUROC $=0.85$ ). Poor outcome was observed in $17 \%$ of the cohort and identified with the CRASH-Basic and IMPACT-Core models to varying degrees: standardized morbidity ratio $=0.89$ vs 0.67 , respectively; calibration $=6.5\left(\chi^{2}\right)$ and 0.59 ( $p$ value) versus $8.52\left(\chi^{2}\right)$ and $0.38(p$ value), respectively; and discrimination $(A \cup R O C)=0.92$ versus 0.83 , respectively.
\end{abstract}

CONCLUSIONS Adult head injury models may be applied with sufficient accuracy to identify predictors of morbidity and mortality in pediatric TBI.

http://thejns.org/doi/abs/10.3171/2016.5.PEDS15427

KEY WORDS brain; injury; acute; prediction; trauma

$\mathrm{T}$ Raumatic brain injury (TBI) remains a major public health problem. ${ }^{6}$ The early management phase of TBI aims to limit secondary insults, achieve hemodynamic stability, obtain accurate neurological assessment, and appropriately select patients for further investigation. ${ }^{10}$ Epidemiological studies demonstrate that the incidence of hospitalization and fatality in TBI is disproportionately high in adolescents. The Centers for Disease Control and Prevention reports more than 1.4 million incidents of TBI in children and in excess of 50,000 deaths annually related to the injury in the US. ${ }^{6}$

Insight into which factors determine prognosis after TBI is useful for clinical practice, research, and making policy. Several steps can be identified in prediction research: univariate analysis, multivariable analysis, and the development of prediction models. ${ }^{15}$ In identifying these steps, several issues need to be addressed, especially selection/coding of predictors of outcome. ${ }^{15}$ Due to the large

ABBREVIATIONS AUROC = area under the receiver operating curve; CRASH = Corticosteroid Randomisation After Significant Head Injury; GCS = Glasgow Coma Scale; GOS = Glasgow Outcome Scale; IMPACT = International Mission on Prognosis and Analysis of randomized Controlled Trials in TBI; IQR = interquartile range; PICU = pediatric intensive care unit; SMbR = standardized morbidity ratio; SMtR = standardized mortality ratio; TBI = traumatic brain injury.

SUBMITTED July 21, 2015. ACCEPTED May 24, 2016.

INCLUDE WHEN CITING Published online August 26, 2016; DOI: 10.3171/2016.5.PEDS15427. 
number of patients required to generate and validate regression models, accurate prediction tools specific to pediatric brain injury are difficult to develop and few have been published. ${ }^{18}$

Prognostic models can be advantageous at both an individual and group level. On an individual level they complement clinical assessment and can be used, albeit with caution, to support decisions on treatment, provide information to relatives, and inform resource allocation. ${ }^{17}$ At a group level, prognostic models can be used to select specific patient subgroups for enrollment in clinical trials or to improve the power of outcome analyses, and importantly, can serve as benchmarks for auditing quality of care. ${ }^{15}$

Baseline predictors include age, sex, mechanism or type of injury, clinical severity, extracranial injury, and the presence of structural abnormalities on neuroimaging., ${ }^{2,5,8,14,17}$ Two prognostic models for use in TBI, developed using large patient databases, have been externally validated: the International Mission on Prognosis and Analysis of randomized Controlled Trials in TBI (IMPACT) model, and the Corticosteroid Randomisation After Significant Head Injury (CRASH) model. ${ }^{16,18}$ Both models have shown good performance in external validations.? Importantly, these models were developed not only to predict mortality but also to predict functional outcome.

Pediatric head injury remains heavily understudied, and many of the current treatment protocols and interventions are derived from evidence in adult studies. ${ }^{3}$ Both IMPACT and CRASH models were independently designed to predict outcomes in adult populations. However, we hypothesize that these models could be of potential value in younger cohorts. In this study we have applied the IMPACT and CRASH prediction models to a pediatric cohort with severe TBI, reviewed over a 5-year period. This cohort has been analyzed to predict both mortality and poor outcome using the extended predictive models.

\section{Methods \\ Study Population}

The data in this study were collected retrospectively from data records of pediatric patients with severe TBI admitted to the pediatric intensive care unit (PICU) of Addenbrooke's Hospital between January 2009 and December 2013.

Inclusion criteria for the study were as follows: 1) confirmed TBI, using CT or MRI; 2) severe injury or failure to demonstrate significant early clinical improvement (i.e., poor neurological condition while withholding sedation) or an injury that required close conservative management or neurological monitoring in the PICU; and 3) patients requiring invasive monitoring of intracranial pressure or arterial blood pressure.

Patients were managed according to current TBI guidelines. ${ }^{1,9}$ Interventions were aimed at keeping intracranial pressure below $20 \mathrm{~mm} \mathrm{Hg}$ using a tiered treatment protocol of positioning, sedation, muscle paralysis, moderate hyperventilation, ventriculostomy, osmotic agents, and induced hypothermia. Clinical outcome was determined using the Glasgow Outcome Scale (GOS). ${ }^{4}$ A good outcome in terms of morbidity was classified as a GOS score of 4 or 5 . The assessments were made on age-dependent milestones.

\section{Data Acquisition}

Patient demographics and 6-month follow-up data were obtained from electronic records. Serum marker levels were extracted from admission blood tests. CT findings were obtained from referring images to the Department of Neurosurgery, Addenbrooke's Hospital. Information on admitting clinical features (such as Glasgow Coma Scale [GCS] score, hypotension, and hypoxia) were obtained from the PICU discharge summary. Outcome measures were obtained from the patients' 6-month clinic follow-up documentation.

\section{Statistical Analysis}

Ordinal data are presented as medians with their interquartile ranges (IQRs) and continuous data as means and associated standard deviations. The individual risk of 14-day mortality was calculated using CRASH-Basic and -CT models, and risk of 6-month mortality calculated with IMPACT-Core and -Extended (including CT findings) models (Table 1). Model accuracy was determined by standardized mortality ratio (SMtR; observed/expected deaths), discrimination was evaluated as the area under the receiver operating curve (AUROC), and calibration assessed using the Hosmer-Lemeshow $\chi^{2}$ test. Statistics were performed in $\mathrm{R}$, in which $\mathrm{p}<0.05$ was considered significant. Results were validated using the online calculators for each test.

\section{Results}

Ninety-four patients with an average age of 7.3 years were admitted to the PICU with a TBI. The baseline clinical features are outlined in Table 2. The modified Marshall scores ranged from 1 to 5 with a mode of 2 . Specific CT features included subarachnoid hemorrhage in $39 \%$ of patients and subdural and extradural hemorrhage in $10 \%$ and $13 \%$, respectively. Of the extraaxial hemorrhages identified, $43 \%$ were evacuated. Petechial hemorrhages were observed in $21 \%$ of the cohort, with obliteration of the basal cisterns also observed relatively infrequently in 10\% of the cohort. Midline shift was observed in 29\% (Table 2). The median ( $\pm \mathrm{IQR})$ blood glucose measurement was $6.8 \pm 1.1 \mathrm{mmol} / \mathrm{L}$ with a median hemoglobin level of $10.5 \pm 1.8 \mathrm{~g} / \mathrm{dl}$.

The mortality rate was $13 \%$ at 14 days and at 6 months. Of those who survived, 63\% had a Glasgow Outcome Scale (GOS) score of 5, $20 \%$ a GOS score of 4 , and $4 \%$ a poor but survivable outcome (3\% a GOS score of $3,1 \%$ a GOS score of 2).

\section{CRASH Discrimination Model}

CRASH-Basic was demonstrated to be a reliable predictor for mortality, in which the SMtR $=1.42$, and both calibration $\left(\chi^{2}=6.1, \mathrm{p}=0.64\right)$ and discrimination (AUROC $=0.92$ ) demonstrated a strong association (Fig. 1A). The CRASH-CT model was also found to show a strong association $\left(\mathrm{SMtR}=0.82\right.$; calibration $=11.84\left[\chi^{2}\right]$ and 0.16 
TABLE 1. Components considered for both CRASH and IMPACT prediction models

\begin{tabular}{ll}
\hline \multicolumn{1}{c}{ Model } & \multicolumn{1}{c}{ Variables } \\
\hline CRASH-Basic & $\begin{array}{c}\text { Age, GCS score, pupils, \& major extracranial } \\
\text { injury }\end{array}$ \\
\hline CRASH-CT & $\begin{array}{c}\text { CRASH-Basic variables, + petechial hemor- } \\
\text { rhage, obliteration of 3rd ventricle/basal } \\
\text { cisterns, SAH, midline shift, \& nonevacuated } \\
\text { hematoma }\end{array}$ \\
\hline IMPACT-Core & $\begin{array}{l}\text { Age, GCS score (motor), pupils } \\
\text { IMPACT-Extended }\end{array}$ \\
$\begin{array}{l}\text { IMPACT-Core variables, + hypoxia, hypotension, } \\
\text { Marshall CT, SAH, \& extradural hematoma }\end{array}$ \\
\hline IMPACT-Lab & $\begin{array}{c}\text { IMPACT-Extended variables, + hemoglobin \& } \\
\text { glucose levels }\end{array}$ \\
\hline
\end{tabular}

$[p$ value]; discrimination $=0.89$ [AUROC]; Fig 1C). The CRASH-Basic model was also able to successfully predict morbidity among patients with a standardized morbidity ratio $\left(\mathrm{SMbR}=0.89\right.$; calibration $=6.5\left[\chi^{2}\right]$ and 0.59 [p value]; discrimination $=0.92$ [AUROC]; Fig. 1B). The only test that failed to provide any predictive accuracy was the application of the CRASH-CT model in predicting poor outcome: $\mathrm{SMbR}=0.55$, calibration $=20.37\left[\chi^{2}\right]$ and 0.01 [ $p$ value]; discrimination $=0.91$ [AUROC]; Fig. 1D).

\section{IMPACT Discrimination Model}

The IMPACT models demonstrated improved ability to discriminate both mortality and morbidity in the pediatric cohort. For mortality, the IMPACT-Core model showed strong correlations $(\mathrm{SMtR}=1.03)$ and was well calibrated $\left(\chi^{2}=8.99, p=0.34\right)$ with good discrimination properties (AUROC $=0.85$; Fig. 2A). The IMPACT-Extended model was also well suited for mortality (SMtR $=1.07$; calibration $=4.67\left[\chi^{2}\right]$ and $0.79[\mathrm{p}$ value $]$; discrimination $=0.87$ [AUROC]; Fig. 3A). Finally, the IMPACT-Lab model demonstrated an SMtR of 0.93, as well as a calibration of $5.44\left[\chi^{2}\right]$ and 0.71 ( $\mathrm{p}$ value), and a discrimination of 0.90 (AUROC; Fig. 3B).

Morbidity was equally well discriminated using the IMPACT models. The IMPACT-Core model demonstrated equally strong correlations $(\mathrm{SMbR}=0.89$; calibration $=8.52\left[\chi^{2}\right]$ and 0.38 [p value $]$; discrimination $=$ 0.83 [AUROC]; Fig. 2B). The IMPACT-Extended model performed similarly $\left(\mathrm{SMbR}=0.89\right.$; calibration $=6.95\left[\chi^{2}\right]$ and 0.54 [p value]; discrimination $=0.87$ [AUROC]; Fig. 3C). Finally the IMPACT-Lab model was also accurate at predicting poor outcome $(\mathrm{SMbR}=0.83$; calibration $=6.57$ $\left[\chi^{2}\right]$ and 0.58 [p value]; discrimination $=0.88$ [AUROC]; Fig. 3D).

\section{Discussion}

In this study we have identified that both the CRASH and IMPACT scoring systems can be applied to a pediatric cohort. The models show positive features that indicate they can discriminate and are largely well calibrated. On this basis they can potentially be applied in pediatrics as long as their shortcomings are acknowledged. Predictive scoring systems are used both clinically and in research
TABLE 2. Patient demographics used to calculate outcome scores

\begin{tabular}{lcc}
\hline \multicolumn{1}{c}{ Variables } & Survivors & Nonsurvivors \\
\hline No. of patients & 82 & 12 \\
\hline Mean age \pm SD (yrs) & $7.3 \pm 5.1$ & $7.5 \pm 5.7$ \\
\hline Males (\%) & $48(59)$ & $8(67)$ \\
\hline Median admission GCS score (range) & $9(3-13)$ & $3(3-9)$ \\
\hline Median motor score (range) & $6(1-6)$ & $1(1-5)$ \\
\hline Pupils (\%) & & \\
\hline Reactive & $74(90)$ & $3(25)$ \\
\hline Fixed unilaterally & $5(6)$ & $3(25)$ \\
\hline Fixed bilaterally & $3(4)$ & $6(50)$ \\
\hline Prehospital hypoxia (\%) & $6(7)$ & $4(33)$ \\
\hline Prehospital hypotension (\%) & $6(7)$ & $2(17)$ \\
\hline Median modified Marshall score & 2 & 3 \\
\hline Subarachnoid hemorrhage on CT (\%) & $28(34)$ & $9(75)$ \\
\hline Epidural mass (\%) & $17(21)$ & $4(33)$ \\
\hline Petechial hemorrhages (\%) & $14(17)$ & $6(50)$ \\
\hline Obliteration of basal cisterns (\%) & $3(4)$ & $6(50)$ \\
\hline Midline shift (\%) & $21(26)$ & $6(50)$ \\
\hline Surgical intervention (\%) & & \\
\hline External ventricular drain & $8(10)$ & $3(25)$ \\
\hline Hematoma evacuation & $15(18)$ & $2(17)$ \\
\hline Decompression & $2(2)$ & $2(17)$ \\
\hline Mean hemoglobin \pm SD (g/dl) & $6.9 \pm 1.9$ & $10.8 \pm 4.1$ \\
\hline Mean glucose \pm SD (mmol/L) & $10.7 \pm 1.8$ & $11.2 \pm 3.4$ \\
\hline
\end{tabular}

to varying degrees. ${ }^{15}$ Clinically, some estimation of prognosis is consciously or subconsciously used by physicians when informing relatives, making treatment decisions, or allocating resources. ${ }^{13}$ In modern-day practice, evidence derived from large data sets is held in greater regard than evidence from the subjective opinion of an individual. The Canadian CT rule and the CHIP (CT in head-injury patients) prediction rule for CT scanning in mild TBI are examples of how prediction models can provide evidence to better inform clinical decisions. ${ }^{19,20}$ Nevertheless, caution is encouraged when outcome prediction models are applied in individual patients; estimates are only probabilities and cannot provide certainty on an actual outcome, particularly neurological. ${ }^{13}$

One of the greatest benefits of prognostic models for outcome in TBI is group stratification in data analysis and enrollment into clinical trials. ${ }^{15}$ Prognostic risk estimation on hospital admission enables populations to be classified according to their prognostic risk distribution..$^{12}$ This is particularly useful in pediatrics given the significant heterogeneity among the cohort. ${ }^{7}$ We can therefore use such models to gain insight into differences in the case mix of different studies.

The application of the CRASH models demonstrated that they were reliable predictors of mortality when both CRASH-Basic and -CT models of the test were applied. Whereas CRASH-Basic was also able to predict morbidity in an accurate fashion, the CRASH-CT test was poorly calibrated by comparison. This is likely to be associated 

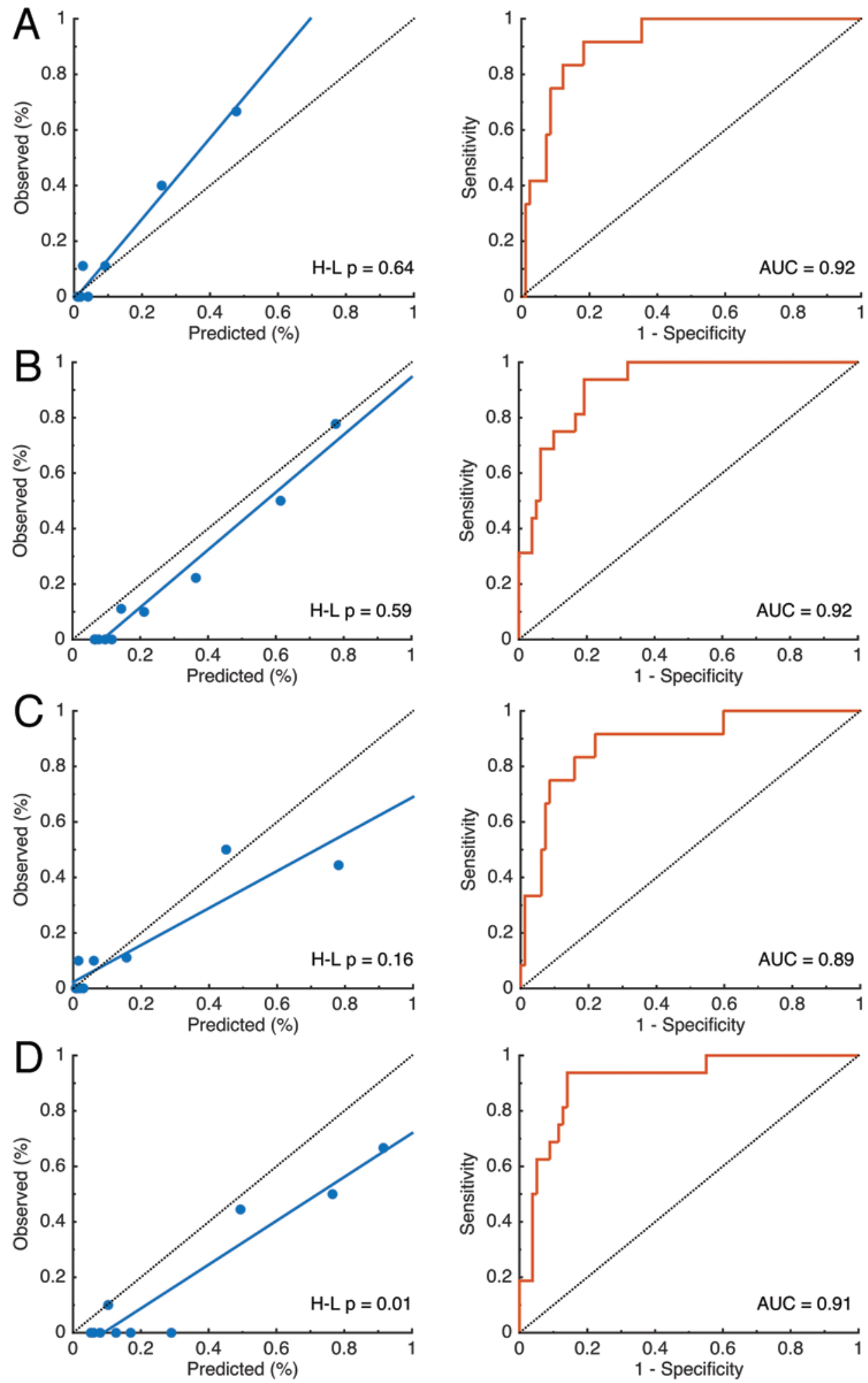

FIG. 1. CRASH model predictions of outcome in children. A: The CRASH-Basic model was demonstrated to be a reliable predictor for mortality, where SMtR $=1.42$ with both calibration $(p=0.64$ ) and discrimination (AUROC $=0.92$ ) demonstrating a strong association with outcome. B: The CRASH-Basic model was also able to successfully predict poor outcome among patients (SMbR $=0.89$, calibration $[\mathrm{p}$ value] $=0.59$, discrimination $[A U R O C]=0.92)$. C: The CRASH-CT model was observed to have a strong association with outcome: $S M t R=0.82$, calibration [p value] $=0.16$, discrimination $[A U R O C]=0.89$. D: The only test that failed to provide any predictive accuracy was the application of the CRASH-CT model in predicting poor outcome, in which the SMbR = 0.55 , calibration $(p$ value $)=0.01$, and discrimination $(A \cup R O C)=0.91$. Figure is available in color online only. 

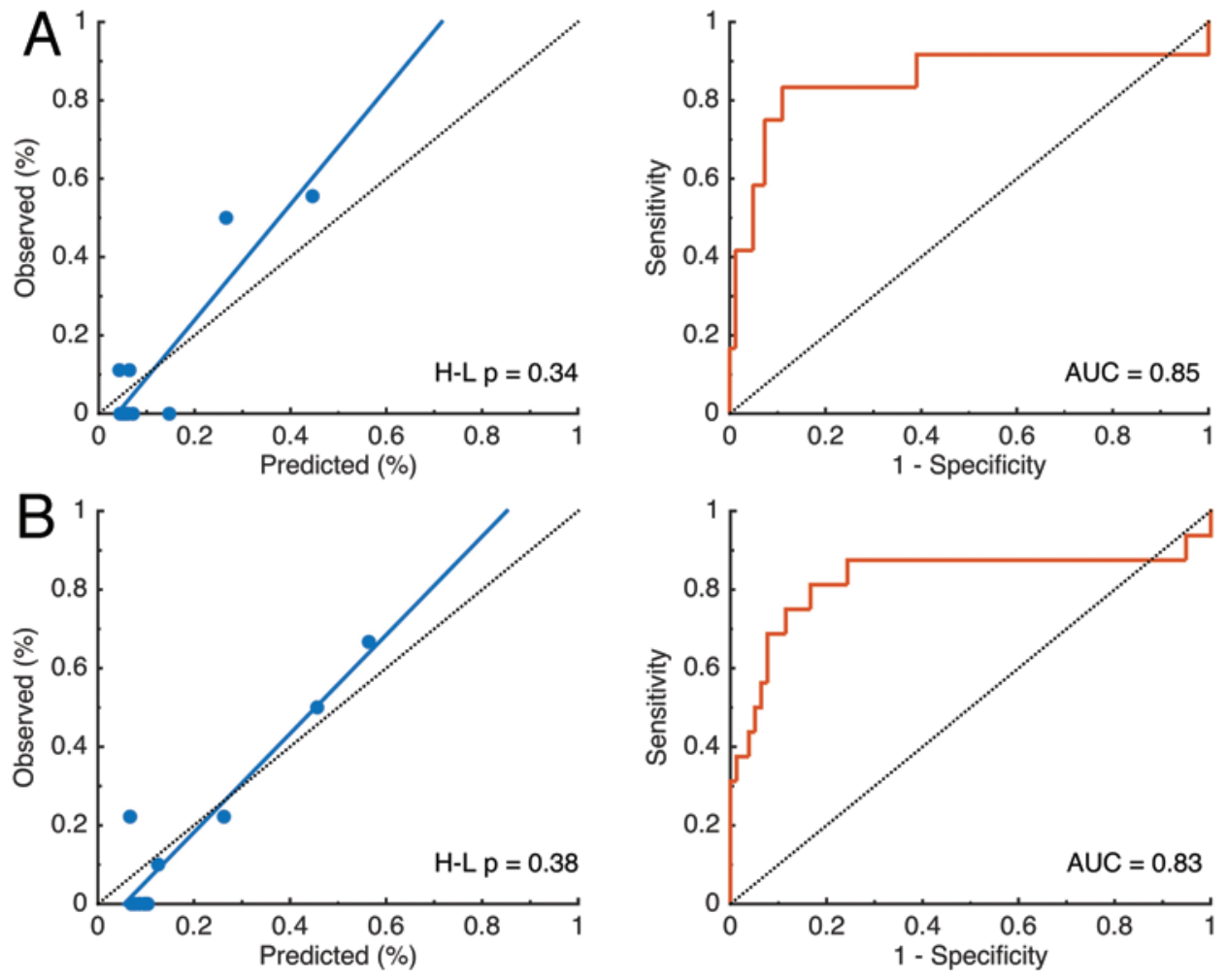

FIG. 2. IMPACT model predictions of outcome in children. A: The IMPACT models demonstrated an improved ability to discriminate both mortality and morbidity in the pediatric cohort. For mortality, the IMPACT-Core model showed strong correlations (SMtR $=1.03$ ) and was well calibrated $(p=0.34$ ) with good discrimination properties (AUROC $=0.85)$. B: Poor outcomes were equally well discriminated using the IMPACT models. The IMPACT-Core model demonstrated equally strong correlations: $\mathrm{SMbR}=0.89$, calibration $(p$ value $)=0.38$, discrimination $(A U R O C)=0.83$. Figure is available in color online only.

with the fact that the CRASH predictive model does not calculate outcomes of patients with a GCS score of $15 .{ }^{18}$ Additionally, in this cohort we are extrapolating below the age of the patient group originally used to create the model. The age range for predicting outcomes in CRASH are collectively grouped for adults under the age of $40 .^{18}$ The inference that adults between the ages of 18 and 40 would have a similar outcome demonstrates that this model in particular is weighted more toward the other variables measured. Given that this is not likely to be the case in a pediatric cohort and that the outcome will very much be age dependent, perhaps improving with increased age and subsequent development, it is reasonable to suspect these factors are responsible for the shortcomings in accuracy.

The IMPACT models performed slightly more favorably overall, with all 3 independently able to predict both morbidity and mortality. IMPACT-Extended and IMPACT-Lab both outperformed IMPACT-Core, which demonstrates the importance of the respective variables on outcome. The IMPACT calculator accounts for ages 14 years and older. The stratification of age into individual years in this manner may account for a better degree of discrimination and fit in these tests.

In this study we have successfully demonstrated that both CRASH and IMPACT analytical models can be used to predict outcomes in morbidity and mortality in a pediatric cohort. This is most applicable for the stratification of patient groups for the analysis of research into pediatric head injury. The pathophysiology of pediatric brain injury is relatively understudied despite large epidemiological studies demonstrating that the incidence of hospitalization and fatal brain injury is disproportionately high in children. ${ }^{11}$ The Centers for Disease Control and Prevention report more than 1.4 million incidents of TBI in children and in excess of 50,000 deaths related to the injury in the US. ${ }^{6}$ Understanding the intricacies of this condition and identifying areas where adult treatments could be used will depend on the careful selection of research groups, a particularly difficult task in pediatric TBI. ${ }^{3}$

There were notable limitations to this study: the retrospective nature increases the possibility of missing data, and the sample size was modest but expected for a single center considering that only intensive care patients were included. To further investigate the findings from this study, a multicenter analysis of pediatric TBI would be beneficial. In this way, new variables could be introduced and less relevant ones excluded in such a way that a more specific model could be adapted. This study has demonstrated proof of concept that these models are appropriate and that this is an endeavor that would be worthwhile in a cohort of severely injured patients. Whether this will be similar in a mild to moderately injured cohort remains unclear.

\section{Conclusions}

Our observations have identified that both the CRASH 

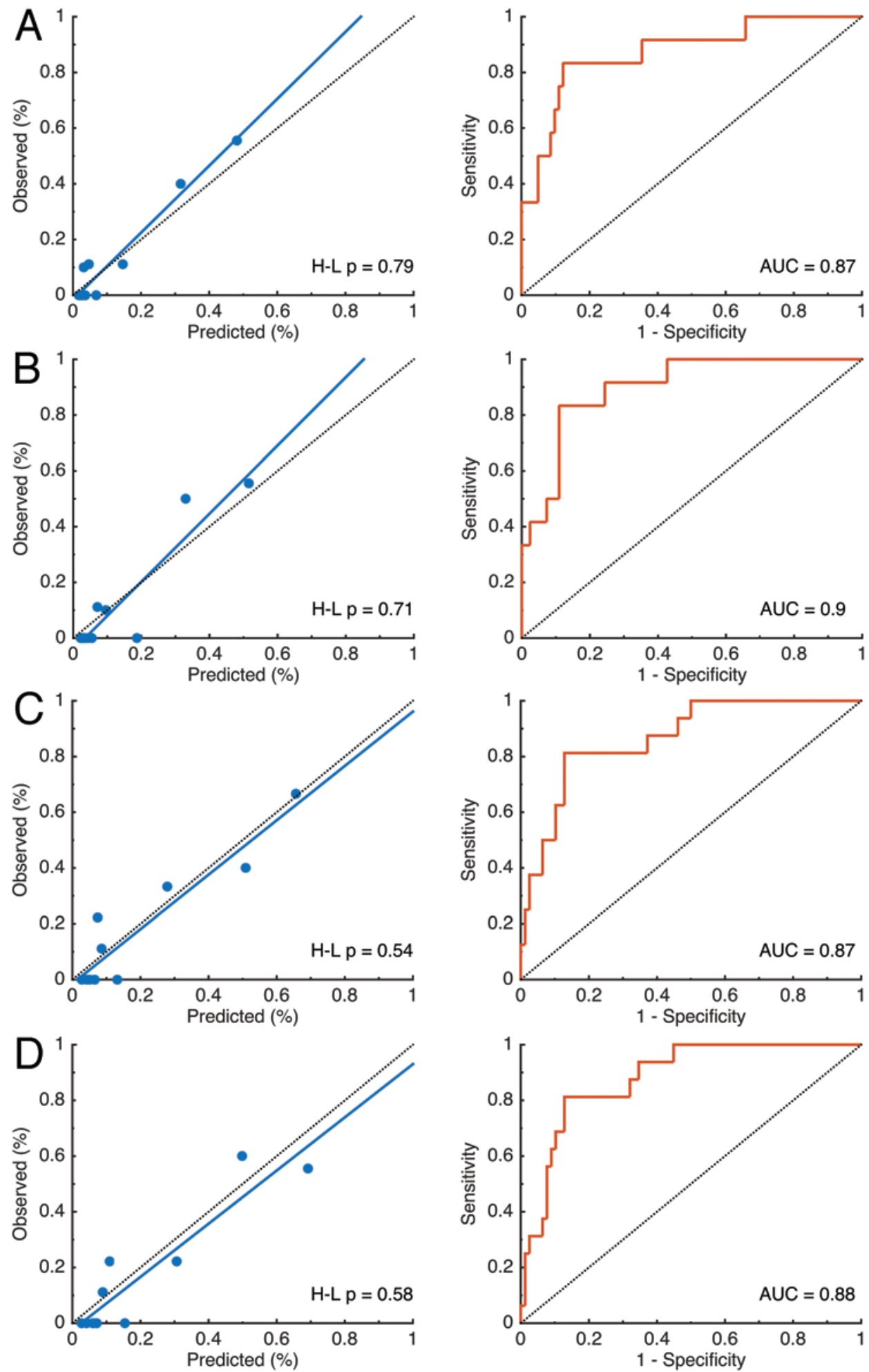

FIG. 3. IMPACT-Extended and -Lab model predictions of outcome in children. A: The IMPACT-Extended model was also well suited for mortality: $S M t R=1.07$, calibration ( $p$ value $)=0.79$, discrimination (AUROC) $=0.87$. B: The IMPACT-Lab model demonstrated an SMtR $=0.93$, calibration of 0.71 ( $p$ value), and discrimination of 0.90 (AUROC). C: The IMPACT-Extended model performed similarly in morbidity: $S M b R=0.89$, calibration ( $p$ value $)=0.54$, discrimination $(A U R O C)=0.87$. D: Finally, the IMPACT-Lab model was also accurate at predicting poor outcome: $S M b R=0.83$, calibration $(p$ value $)=0.58$, discrimination $(A U R O C)=0.88$. Figure is available in color online only. 
and IMPACT scoring systems can be applied to a pediatric cohort. As such, the outcome of pediatric head injury can be predicted with excellent discrimination and good fit using parameters available at the bedside. In an understudied patient group this will allow for improved group stratification in data analysis and enrollment into clinical trials. Although this study is limited by its modest sample size, it acts as a proof of concept model that these analytical tools can be applied to pediatric patients with TBI. Nevertheless, due to the sample size in the current cohort, external validation would be appropriate with a multicenter data set.

\section{Acknowledgments}

We gratefully acknowledge financial support as follows. Research support was provided by the Medical Research Council (grant nos. G0600986 ID79068 and G1002277 ID98489) and the National Institute for Health Research Biomedical Research Centre Cambridge (Neuroscience Theme, and Brain Injury and Repair Theme). Dr. Hutchinson is supported by an NIHR Research Professorship and an Academy of Medical Sciences/Health Foundation Senior Surgical Scientist Fellowship.

\section{References}

1. Adelson PD, Bratton SL, Carney NA, Chesnut RM, du Coudray HE, Goldstein B, et al: Guidelines for the acute medical management of severe traumatic brain injury in infants, children, and adolescents. Chapter 3. Prehospital airway management. Pediatr Crit Care Med 4 (3 Suppl):S9-S11, 2003

2. Bouras T, Stranjalis G, Korfias S, Andrianakis I, Pitaridis M, Sakas DE: Head injury mortality in a geriatric population: differentiating an "edge" age group with better potential for benefit than older poor-prognosis patients. J Neurotrauma 24:1355-1361, 2007

3. Brady KM, Shaffner DH, Lee JK, Easley RB, Smielewski P, Czosnyka M, et al: Continuous monitoring of cerebrovascular pressure reactivity after traumatic brain injury in children. Pediatrics 124:e1205-e1212, 2009

4. Brooks DN, Hosie J, Bond MR, Jennett B, Aughton M: Cognitive sequelae of severe head injury in relation to the Glasgow Outcome Scale. J Neurol Neurosurg Psychiatry 49:549-553, 1986

5. Coronado VG, Thomas KE, Sattin RW, Johnson RL: The CDC traumatic brain injury surveillance system: characteristics of persons aged 65 years and older hospitalized with a TBI. J Head Trauma Rehabil 20:215-228, 2005

6. Faul M, Xu L, Wald MM, Coronado VG: Traumatic Brain Injury in the United States: Emergency Department Visits, Hospitalizations, and Deaths. Atlanta: Centers for Disease Control and Prevention, National Center for Injury Prevention and Control, 2010

7. Han J, King NK, Neilson SJ, Gandhi MP, Ng I: External validation of the CRASH and IMPACT prognostic models in severe traumatic brain injury. J Neurotrauma 31:1146-1152, 2014

8. Hukkelhoven CW, Steyerberg EW, Rampen AJ, Farace E, Habbema JD, Marshall LF, et al: Patient age and outcome following severe traumatic brain injury: an analysis of 5600 patients. J Neurosurg 99:666-673, 2003

9. Kochanek PM, Carney N, Adelson PD, Ashwal S, Bell MJ, Bratton S, et al: Guidelines for the acute medical management of severe traumatic brain injury in infants, children and adolescents - second edition. Pediatr Crit Care Med 13 (Suppl 1):S1-S82, 2012

10. Kolias AG, Guilfoyle MR, Helmy A, Allanson J, Hutchinson PJ: Traumatic brain injury in adults. Pract Neurol 13:228235,2013

11. Langlois JA, Rutland-Brown W, Thomas KE: Traumatic Brain Injury in the United States: Emergency Department Visits, Hospitalizations, and Deaths. Atlanta: Centers for Disease Control and Prevention, National Center for Injury Prevention and Control, 2004

12. Lingsma HF, Maas AIR, Steyerberg EW: [Prognostication of moderate and severe traumatic brain injury.] Ned Tijdschr Geneeskd 154:107-114, 2010 (Dutch)

13. Lingsma HF, Roozenbeek B, Steyerberg EW, Murray GD, Maas AI: Early prognosis in traumatic brain injury: from prophecies to predictions. Lancet Neurol 9:543-554, 2010

14. Livingston DH, Lavery RF, Mosenthal AC, Knudson MM, Lee S, Morabito D, et al: Recovery at one year following isolated traumatic brain injury: a Western Trauma Association prospective multicenter trial. J Trauma 59:1298-1304, 2005

15. Maas AI, Lingsma HF, Roozenbeek B: Predicting outcome after traumatic brain injury. Handb Clin Neurol 128:455474,2015

16. Maas AI, Murray GD, Roozenbeek B, Lingsma HF, Butcher I, McHugh GS, et al: Advancing care for traumatic brain injury: findings from the IMPACT studies and perspectives on future research. Lancet Neurol 12:1200-1210, 2013

17. Mitra B, Cameron PA, Gabbe BJ, Rosenfeld JV, Kavar B: Management and hospital outcome of the severely head injured elderly patient. ANZ J Surg 78:588-592, 2008

18. Perel P, Arango M, Clayton T, Edwards P, Komolafe E, Poccock $\mathrm{S}$, et al: Predicting outcome after traumatic brain injury: practical prognostic models based on large cohort of international patients. BMJ 336:425-429, 2008

19. Smits M, Dippel DW, Steyerberg EW, de Haan GG, Dekker $\mathrm{HM}$, Vos PE, et al: Predicting intracranial traumatic findings on computed tomography in patients with minor head injury: the CHIP prediction rule. Ann Intern Med 146:397-405, 2007

20. Stiell IG, Wells GA, Vandemheen K, Clement C, Lesiuk H, Laupacis A, et al: The Canadian CT Head Rule for patients with minor head injury. Lancet 357:1391-1396, 2001

\section{Disclosures}

The authors report no conflict of interest concerning the materials or methods used in this study or the findings specified in this paper.

\section{Author Contributions}

Conception and design: Young, Guilfoyle. Acquisition of data: Young. Analysis and interpretation of data: Young, Guilfoyle, Hutchinson. Drafting the article: Young. Critically revising the article: Guilfoyle, Fernandes, Garnett, Agrawal, Hutchinson. Reviewed submitted version of manuscript: all authors. Approved the final version of the manuscript on behalf of all authors: Young. Study supervision: Fernandes, Garnett, Agrawal, Hutchinson.

\section{Correspondence}

Adam Young, Division of Academic Neurosurgery, Department of Clinical Neurosciences, Addenbrooke's Hospital, University of Cambridge, Cambridge, United Kingdom. email: ay276@cam. ac.uk. 